\title{
The Cepheid Data Base
}

\author{
L. N. Berdnikov \\ Sternberg Astronomical Institute, 13 Universitetskij prosp., Moscow \\ 119899, Russia
}

\begin{abstract}
Since 1981, we have been regularly carrying out photoelectric observations of Cepheids. By now nearly 20450 observations in UBVRI have been acquired. These observations are the basis of our Cepheid data base; presently we have collected on magnetic media near 88000 photoelectric as well as near 18000 radial velocity measurements. We shall continue to append to this Cepheid data base our new observations as well as published data.
\end{abstract}

\section{Introduction}

It is very important today to have the photometric and radial velocity measurements of Cepheid variables in electronic form. These data can be used for study of the Cepheids themselves as well as for study of the Galaxy structure.

We began the work of creation of Cepheid data base in 1980 .

\section{Structure of Cepheid data base}

The collection of the observational data is managed in two ways. Firstly, there are photoelectric data on the Cepheids observed by us, and secondly, published photoelectric observations and radial velocity measurements are collected. At the present moment nearly 20450 observations in UBVRI system have been obtained, and the information from 442 published papers has been gathered.

Both the photoelectric observations and radial velocities catalogues have been converted to machine readable form. The first catalogue contains nearly 88000 photoelectric observations obtained for the both classical and type W Vir Cepheids and several related stars in various photometric systems, while the second one includes approximately 18000 radial velocity measurements.

Also, there is a machine readable version of the reference - bibliographic catalogue, which consists of two files. The one file contains bibliographic information on publications accessible to us which are placed in alphabetical order of the authors. There is following information for each paper: the first line is the key words one beginning with symbols "******"; the second line contains the paper's number, authors and year of the publication; the third line is the paper's title; the fourth line contains the reference. If there are rows of continuing characters they are shifted on two positions to right. For example: 
****** CEP PHE ABUND

73. Diethelm R., 1986.

Physical parameters of pulsating variables with periods between one and three days. I. Photometry and metallicities.

Astron. and Astrophys. Suppl., 1986, v.64, p.261-274.

The other file contains the cross-referencing in the papers Cepheid variable names, placed after paper's number. This catalogue allows one to find the necessary literature by key words and/or by Cepheid's name.

Moreover, a series of FORTRAN programs has been written, which are used for modernisation and maintenance of the catalogues data.

\section{Discussion}

The Cepheid data bank is used in a series of the Cepheid studies. Some results had already been published, for example: "The catalogue of light curves parameters, distances and space coordinates of classical Cepheids" (Berdnikov, 1987a), "The distribution of Cepheids in the Galaxy" (Berdnikov, 1987b), "The groupings of the Cepheids in the Galaxy" (Berdnikov, Efremov, 1989), "Light curves and stability of the periods of the components of CE Cassiopeiae" (Berdnikov, 1990), "Light curves of double-mode Cepheids" (Berdnikov, 1992), "Isolines of surface density and z-coordinates of Cepheids" (Berdnikov, Efremov, 1993), "A study of pulsation stability of seven northern Cepheids with the longest periods" (Berdnikov,1994), "A study of period changes of small amplitude Cepheids in Aql, CMa, Car and Cas" (Berdnikov L.N., Pastukhova E.N., 1994a), "A study of period changes of small amplitude Cepheids in Cen, Cru, Cyg, Gem, Lac, Lyr, Mon, Nor and Oph" (Berdnikov L.N., Pastukhova E.N., 1994b), etc.

At present, a new version of the atlas of light and colour curves of the all photoelectrically observed in UBVRI system Cepheids has been created.

In the near future it is planned to create the Cepheid light curves atlas for all filter-measurements in all photometric systems.

We shall continue to append our Cepheid data base with our new observations as well as with published data.

\section{References}

Berdnikov, L.N. 1987a, Peremennye Zviozdy, 22, 505

Berdnikov, L.N. 1987b, Pis'ma Astron. Zh., 13, 110

Berdnikov, L.N. 1990, AZh, 67, 798

Berdnikov, L.N. 1992, Pis'ma Astron. Zh., 18, 654

Berdnikov, L.N. 1994, Pis'ma Astron. Zh., 20, 285

Berdnikov, L.N., \& Efremov, Yu.N. 1989, AZh, 66, 537

Berdnikov, L.N., \& Efremov, Yu.N. 1993, Pis'ma Astron. Zh., 19, 957

Berdnikov, L.N., \& Pastukhova, E.N. 1994a, Pis'ma Astron. Zh., 20, 567

Berdnikov, L.N., \& Pastukhova, E.N. 1994b, Pis'ma Astron. Zh., 20, 829 